\title{
Interactive Learning of Data Structures and Algorithmic Schemes ${ }^{\star}$
}

\author{
Clara Segura, Isabel Pita, Rafael del Vado Vírseda, \\ Ana Isabel Saiz, and Pablo Soler \\ Departamento de Sistemas Informáticos y Computación \\ Universidad Complutense de Madrid, Spain \\ \{csegura, ipandreu,rdelvado\}@sip.ucm.es, \\ \{anussita,ileras\}@gmail.com
}

\begin{abstract}
We present an interactive environment called Vedya for the visualization of data structures and algorithmic schemes which can be used as a very useful educational tool in Computer Science. The integration of Vedya and the Virtual Campus of the Complutense University of Madrid has allowed us to manage the whole administration of the individual students' homework, including generating exercises, tests, grading delivered homework, and storing the achieved results. The part of the system concerning data structures has been evaluated during the last academic course 2006/07. By means of the Vedya tool, the students benefited from complementary and interactive material, facilitating the intuitive comprehension of most typical operations of classical data structures without any restriction of time or material.
\end{abstract}

\section{Introduction}

We present an interactive environment tool called Vedya for the visualization of data structures and algorithmic schemes. The pedagogical aim of Vedya is to facilitate the student's grasp of the target procedures of education in Computer Science by means of interactive learning, in order to facilitate teamwork and communication between teachers and students.

For this purpose, we have integrated Vedya in a motivating environment such as the Virtual Campus of the Complutense University of Madrid (https://www. ucm.es/info/uatd/CVUCM/index.php) facilitating the accessibility, understanding and visualization of the main data structures and algorithmic schemes. The combination of the Vedya tool and the Virtual Campus has allowed us to control the whole administration of the individual students' homework including generating exercises, tests, grading delivered homework, and storing the achieved results. By means of the Vedya tool, the students have benefited from complementary and interactive material, facilitating the intuitive comprehension of most typical operations of classical data structures without restrictions of time or material.

\footnotetext{
* The authors have been partially supported by the Spanish National Projects MERITFORMS (TIN2005-09027-C03-03) and PROMESAS-CAM (S-0505/TIC/0407).
} 
Moreover, the continuous utilization of the tool during the theoretical classes of the second four-month period has allowed us to reach one of the most useful educational aims in Computer Science in order to settle some of the academic deficiencies of this kind of subjects: to support the continuous, personal and interactive work across a virtual classroom.

During the academic course 2006/07, Vedya has been freely accessible across the Virtual Campus. In this time, and within the framework of an Educational Innovation Project in Computer Science, we have evaluated the part of the tool dedicated to the study of the main data structures that provides interactive learning support to guide our students in their comprehension of a modern (imperative or declarative) programming language. At this moment, the tool has been widely used to illustrate in a graphical, visual and intuitive way the following well-known data structures [1]: linear data structures (stacks and queues), tree-like data structures (binary search trees, AVL trees, and heaps) and functional data structures (ordered and hash tables). Additionally, the flash animations incorporated in the tool have been used to illustrate other data structures like red-black trees, 2-3-4 trees and graphs; and to show how data structures are used to solve problems. Thanks to this effort, our students have assimilated, inside a motivating framework, one of the fundamental concepts of the subject, the difference between the formal description of the behavior of the data structures provided by the algebraic specification [2], and their implementation using a concrete programming language.

The outline of the paper is the following. In Section 2 we describe Vedya from the point of view of the tool's user. In Section 3 the implementation is explained. Section 4 provides the results obtained from the application of the tool in the last academic course. Finally, in Section 5 we conclude and outline future work.

\section{The Vedya Tool: Description}

Vedya is an interactive environment for learning data structures and algorithms. It covers the most common data structures: stacks, queues, binary search trees, AVL trees, priority queues, and sorted and hash tables. Moreover, it also provides other different types of data structures, like one for an implementation of a doctor's office. Concerning the algorithmic schemes, it covers the most common resolution methods [4: greedy, divide and conquer, dynamic programming, backtracking, and branch and bound.

Lots of work has already been done on data structures and algorithm visualization. However, usually, tools are not complete, have a lack of common Graphical User Interface or can only be executed over some operating systems. In [5] Chen and Sobh present a tool for data structure visualization and userdefined algorithm animation. The data structures available are arrays, stacks, queues, binary search trees, heaps and graphs. The most relevant improvement of this tool is the possibility of executing user-defined algorithms and visualize the state of the data structures used by the algorithms. 


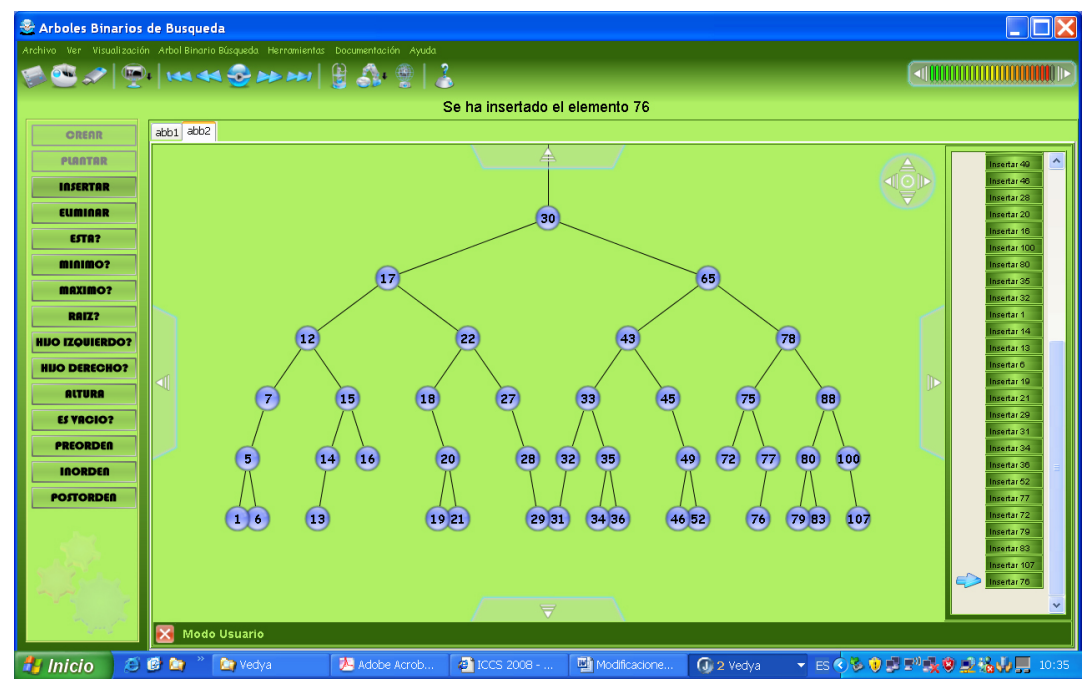

Fig. 1. Main window for the data structures part of the Vedya tool

Nevertheless, Vedya is something more than a data structure execution tool. The main features that differentiate it from other interactive tools are:

(1) First of all, Vedya is created to supply the students with a tool that facilitates the study of both data structures and algorithms. Therefore, all the data structures and algorithmic methods taught in our courses are integrated in the same environment. The environment provides a lot of facilities to make executions more user-friendly: Vedya can be executed using Sun's j2re 1.4.2.xx. It allows the execution of several data structures/algorithms and several sequences of operations on the same structure at the same time making use of a multi-windows and multi-frame system. It also allows saving sequences of operations in order to continue the execution later on. Operations in a sequence can be deleted and added.

(2) Vedya offers several learning possibilities. The main one is the interactive execution of data structures and algorithms, but it is also possible to create simulations that execute automatically, visualize tutorials, and solve tests within the same environment. It also integrates a set of animations that show how data structures are used to solve some problems.

(3) Concerning the data structures part, Vedya offers different views for the data structure behavior and the data structure implementations. Moreover, in most of the data structures both a static and a dynamic implementation are shown. Operations may be executed in any view, and the user can move from one view to another to see the changes in any moment.

(4) The following algorithms are implemented:

- divide and conquer: binary search and quicksort,

- dynamic programming: knapsack problem, 
- greedy method: non-fractional knapsack problem, Dijkstra's algorithm,

- branch and bound: knapsack problem.

The students can visualize how different algorithmic schemes are applied to solve the same problem and under which conditions they can be used.

(5) The environment integrates documentation related to the algebraic specification, the implementation code and the operation cost of each data structure/algorithm.

Currently there exist two versions of the tool. The old version contains all the data structures and algorithmic schemes mentioned above while the new one offers a subset of them in a more attractive visual environment. Figures in this paper correspond to the new version, which can be found at http://www.fdi.ucm. es/profesor/csegura/.

\subsection{Tool Usage}

When the application is started the user selects one area: data structures or algorithmic schemes, and chooses a particular one. Then, the main window for the selected data structure or algorithmic scheme is opened. For all cases this window looks similar. The central panel is used to represent the data structure, on the left there is a list of the actions that can be executed. The allowed actions are highlighted while the non-allowed ones are disabled. The panel on the right shows the actions that have already been executed on the data structure.

On Fig. 11 we show an example of the main window for binary search trees. The selected key type is string, but the system also allows char and string types. On this tree, the user may continue executing actions using the left hand operations panel, or he can use the simulation facilities of the menu to go up on the sequence of actions to see previous states or restart the sequence from the beginning. The central panel offers some tree drawing facilities that allows the user to expand and contract the tree, as well as to move over the screen to see the hidden parts. Notice also that just above the central panel the result of the last action is shown.

On the top of the screen there is a menu that facilitates managing the system. We can create a new data structure; open an existing one; or save the state of the editing one. We can change the view, from behavior, that is the one by default, to implementation, either static or dynamic. On Fig. 2 we can see first the behavior view of a queue. When we insert an item, a truck throws it on the top of the maze. Then, the item falls down until it finds the end of the maze or a previous one. When we extract an item, the end of the maze opens and the first item falls down. The use of the maze illustrates that items cannot jump over the previous ones and the fact that in a queue items are extracted in the order that they come in. Below the behavior view we have the representation of the static implementation based on a circular array and a dynamic implementation based on pointers. On the static implementation, the array is represented by a circle where items are inserted and extracted counterclockwise. This representation stresses the fact that there is not a final element of the array and also that we 

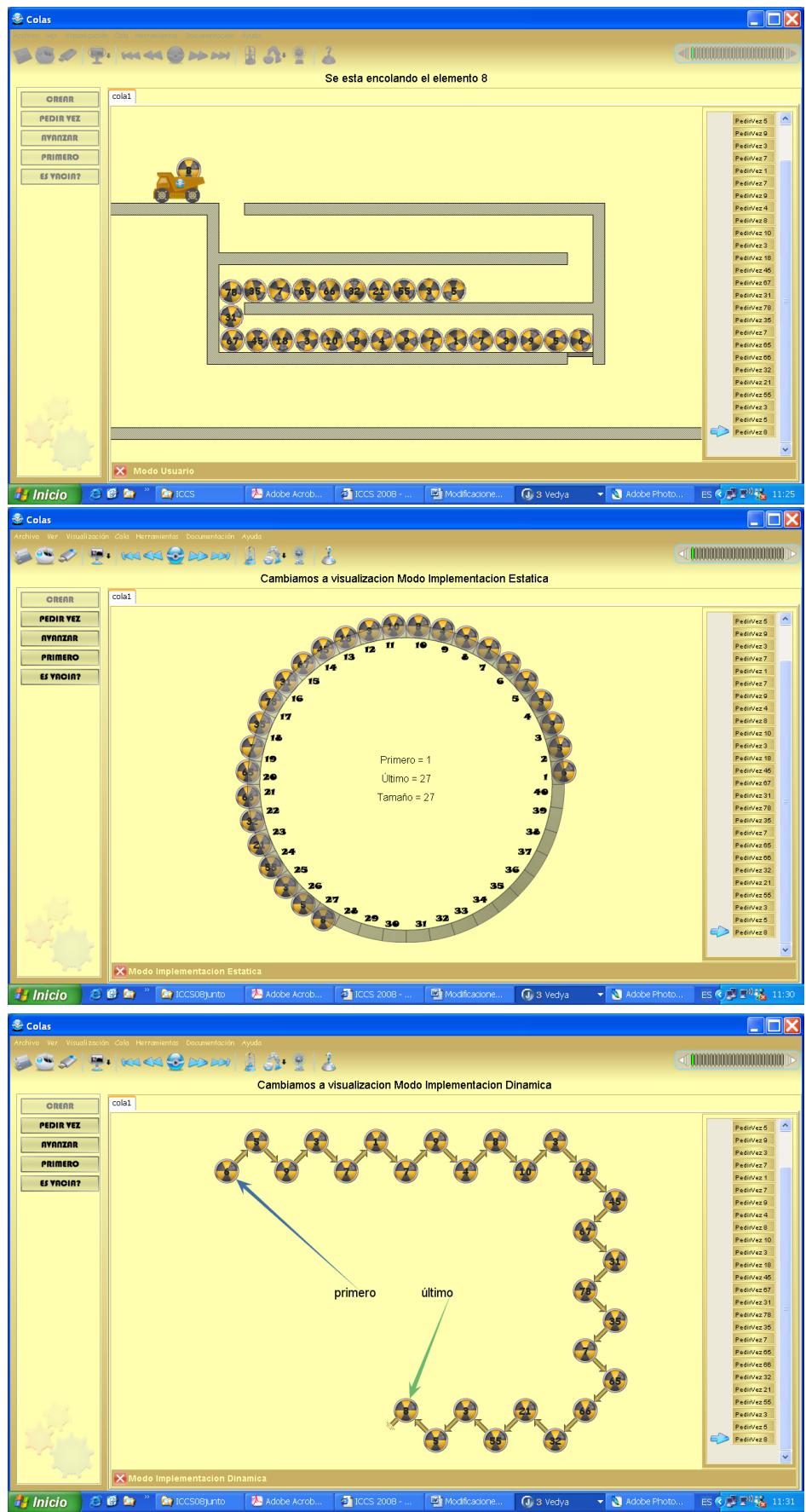

Fig. 2. Behavior and implementations views of queues 
can always insert an item unless the array is completely full. On the dynamic implementation we use short arrows to represent the pointers between the items. Two long arrows point to the first item (written as primero in the figure) and to the last one (written as ultimo). The sequence of modifications are shown step by step so that students notice its relevance in order not to lose any pointer.

Using the menu we can also execute the operations on the data structure, use the simulation facilities and change the execution speed. In the documentation part the user can consult some documentation about the data structure, like the algebraic specification, the implementation code and the cost of each implementation, and finally it can change to the associate Vedya-test tool, where the user can do some proposed test about the selected data structure.

The main window for the execution of algorithmic schemes looks similar. There are panels for drawing the execution of the algorithm, for introducing the input data and for showing the actions being executed. The simulation facilities are also available.

\subsection{Data Types Animations}

Vedya is complemented with a set of tutorials on data types and a set of algorithm animations showing the usage of a particular data type to solve a given problem. This set of tutorials and algorithm animations are developed in Flash, and can be accessed independently from Vedya's initial menu. We have tutorials for stacks, queues, binary search trees, red-black trees and priority queues. We have a tutorial about the heap-sort algorithm, an animation of the insertion in a 2-3-4 tree, and examples of:

- The use of stacks to evaluate an expression in postfix form, or to transform an infix expression to a postfix one.

- The use of queues to obtain the breath-first tree traversal.

- The use of stacks, queues and double queues to check palindromes.

Finally, we have some animations on graphs: to obtain the minimum spanning tree using the Prim and Kruskal algorithms and to compute minimum paths using the Dijsktra algorithm 6].

\subsection{The Vedya-test Tool}

As we have said Vedya also offers facilities to solve tests about the data structures and algorithms that are being studied. The Vedya-test tool can be invoked from the Vedya tool, or it can be executed independently. The tool offers facilities to teachers that allow them to create/modify/delete questions in a database, and to create tests from the database of questions. The student visualizes the tests, solves them and obtains the solutions. Questions are grouped by subject on the database, but it is possible to mix questions about different data structures in the same test. 


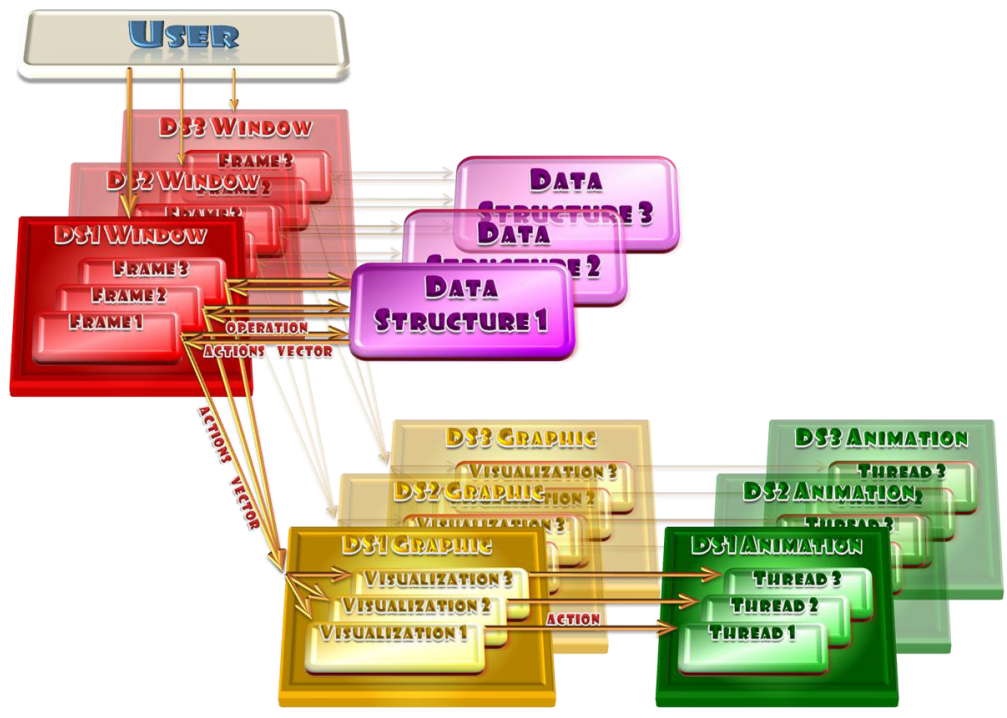

Fig. 3. Tool design

\section{The Vedya Tool: Implementation}

One of the main objectives when Vedya was implemented was that its extension with new data structures or algorithmic schemes should be as easy as possible. The main feature of the implementation is that both the data structures and the algorithms being represented are actually implemented in the tool separately from their graphical representations. The design, shown in Fig. 3, is modularly divided into four blocks: interface, graphics, threads and implementations of the data structures and algorithms. This means that whenever we desire to change a graphical representation we do not need to change the data structure or the algorithm itself. The windows and frame act as communication channel between implementation and the graphics. The threads only communicate with the graphics. Inheritance is exploited in order to reuse as much code as possible. The different blocks are communicated by means of "actions":

- When the user executes an operation in the interface over a data structure or executes an algorithm, the frame sends it to the implementation of the data structure/algorithm to be executed.

- As a result of such execution, a vector of atomic actions to be graphically represented in an animated way is returned to the frame. For example, when inserting an element in a binary search tree, information about the path followed by the element being inserted is returned.

- The frame sends the vector of actions to the graphical module, which paints the necessary elements and sends to the threads block each action to be animated, depending on the kind of selected visualization (user, static implementation or dynamic implementation). 


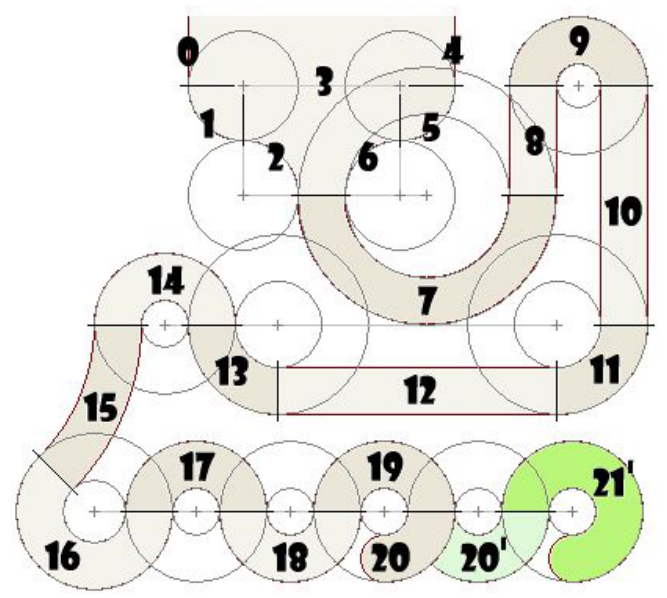

Fig. 4. Stack tracks design

Java threads are used in order to animate operations on data structures. Each operation divides into actions and the animation sequence of each action is divided into tracks where different kinds of movements are applied: circular, radial, horizontal, vertical or point to point. In Fig. 4 we show the circular tracks for the stack user representation, which consists of a dead-end pipe.

\section{Interactive Learning}

In order to obtain a detailed evaluation of the usage of Vedya, we have proposed several tests related to the behavior, implementation and application of the main data structures offered by the tool. We also collect students' opinion using Vedya in the Data Structures course at the second year, and in the Programming Methodology and Technology course at the third year, respectively.

The vast majority of our engineering and computer science students have taken an introductory programming course in the first academic year, typically in Pascal. Although the learning of the main algorithmic schemes and programming techniques is not a prerequisite to the subject of Data Structures, many students elect to take it either prior to, or concurrent with, Programming Methodology and Technology. As a result, although a pseudocode programming language for Data Structures is the assumed language for Data Structures, many students have enough knowledge about $\mathrm{C}++$ or Java programming languages through the integrated programming laboratories of parallel courses and subjects.

Taking into account this profile, skills and background of our students, we have proposed 8 tests in the Virtual Campus of the Complutense University of Madrid. The number of engineering students registered in the Virtual Campus was just over 320 distributed in three groups (130 in group A, 59 in group B, and 131 in group C). Table 1 shows the number of the students who answered each of the tests in the corresponding group. 
Table 1. Students answering the tests

\begin{tabular}{|c|c|c|c|c|c|c|c|c|}
\hline & Stacks 1 & Stacks 2 & Queues & Sequences & BST & AVL & RB & Heaps \\
\hline Group A (130) & 61 & 50 & 45 & 32 & 37 & 34 & 41 & 38 \\
\hline Group B (59) & 26 & 23 & 23 & 19 & 18 & 17 & 17 & 18 \\
\hline Group C (131) & 59 & 44 & 37 & 24 & 36 & 45 & 32 & 28 \\
\hline Total & 147 & 118 & 105 & 75 & 91 & 96 & 90 & 76 \\
\hline
\end{tabular}

We observe that, from the second test on, the number of students becomes stable in a number lightly low to the number of students who access regularly to the Virtual Campus. These numbers, though seemingly high, are only between $23 \%$ (75 students of 320 ) and $37 \%$ (118 of 320) of registered students, which shows the high rate of students giving up in this topic from the beginning.

Table 2. Percentage of correct answers

\begin{tabular}{|l|c|c|c|c|c|c|c|c|}
\hline & Stacks 1 & Stacks 2 & Queues & Sequences & BST & AVL & RB & Heaps \\
\hline Group A & $76.4 \%$ & $82.5 \%$ & $77.8 \%$ & $65.6 \%$ & $82.2 \%$ & $84.9 \%$ & - & $86.3 \%$ \\
\hline Group B & $78.9 \%$ & $83.6 \%$ & $85.0 \%$ & $63.6 \%$ & $86.2 \%$ & $87.7 \%$ & $90.9 \%$ & $90.2 \%$ \\
\hline Group C & $76.2 \%$ & $79.8 \%$ & $73.5 \%$ & $69.0 \%$ & $83.5 \%$ & - & $68.9 \%$ & $86.8 \%$ \\
\hline
\end{tabular}

Table 2 shows the percentage of correct answers in the three groups: In general, it is high, which demonstrates the interest of the students who have taken part. In group B, the percentage is slightly higher than groups A and C; since $85 \%$ of the students who have decided to complete the tests across the Virtual Campus of group B are not "new" students of this subject.

Table 3. Comparison of academic results with previous courses

\begin{tabular}{|c|c|c|c|c|c|}
\hline & $\mathbf{2 0 0 2 / 0 3}$ & $\mathbf{2 0 0 3 / 0 4}$ & $\mathbf{2 0 0 4 / 0 5}$ & $\mathbf{2 0 0 5 / 0 6}$ & $\mathbf{2 0 0 6 / 0 7}$ \\
\hline Not attended & $57.6 \%$ & $45.3 \%$ & $42.3 \%$ & $64.7 \%$ & $50.8 \%$ \\
\hline Passed & $15.3 \%$ & $22.2 \%$ & $20.2 \%$ & $18.2 \%$ & $30.1 \%$ \\
\hline Failed & $27.1 \%$ & $32.5 \%$ & $37.5 \%$ & $17.1 \%$ & $18.9 \%$ \\
\hline
\end{tabular}

Table 3 shows the percentage of students that did not attend the final exam, those who passed, and those who failed during the last five years. We observe that in the last course, in which we have applied the Vedya tool, we have reduced by $14 \%$ the percentage of students giving up the course with respect to the previous course, and at the same time, we have increased by $12 \%$ the percentage of students that passed the exam. The percentage of students that failed the exam increased by $2 \%$ due to the rise of students attending the exam. Comparing with previous courses (2003 to 2004) the percentage of students that passed has increased between $8 \%$ (with respect to the course 2003/04) and 15\% (with respect to the course $2002 / 03)$. 


\section{Conclusions and Future Work}

In this paper, we have presented a novel interactive tool called Vedya for the visualization of data structures and algorithmic schemes which can be used as a very useful educational tool to aid first-year engineering and computer science students learn Data Structures and Algorithms. The main benefit of this kind of software is to facilitate the student's grasp of the target procedures of education, to facilitate teamwork and communication between teachers and students. In this sense, the novel integration of the Vedya tool in the virtual classroom has allowed us to motivate the participation of the students, and to obtain one of the most powerful goals from the educational viewpoint. Furthermore, the personalization and automatization of the learning process has avoided the lack of motivation for the abstract subjects in Computer Science because students find them 'useful'. The fact that interface language is Spanish is for our students one additional reason to find the environment more friendly. Finally, it is frequent that a tool is useful not only to the original purpose it was created for, but also for other subsidiary (though not less important) uses. In this sense, we believe that the development of this tool can help educators to use the Vedya tool directly or to create other similar tools.

As future work, we plan to develop alternative ways to integrate the Vedya tool in a Virtual Campus based on WebCT. We are interested in the application of the tool and the interactive learning methodology presented in this paper inside of different models of virtualization and e-learning in Computational Science: the development of digital repositories of Learning Objects about data structures and algorithmic schemes in the Vedya tool using IMS DRI and Moodle, the integration of the Vedya tool in an Intelligent Tutorial System, or the application of typical tools of the Web 2.0 philosophy.

\section{References}

1. Weiss, M.: Data Structures and Problem Solving Using Java. Addison-Wesley, Reading (1998)

2. Martí, N., Ortega, Y., Verdejo, A.: Estructuras de datos y métodos algorítmicos: ejercicios resueltos. Prentice-Hall, Englewood Cliffs (2003)

3. Peña Marí, R.: Diseño de programas. Formalismo y abstracción, 3rd edn. PrenticeHall, Englewood Cliffs (2005)

4. Cormen, T.H., Leiserson, C.E., Rivest, R.L.: Introduction to Algorithms. The MIT Press/McGraw-Hill (2001)

5. Chen, T., Sobh, T.: A tool for data structure visualization and user-defined algorithm animation. In: Frontiers in Education Conference (2001)

6. Pita, I., Segura, C.: A tool for interactive learning of data structures and algorithms. In: 8th International Symposium on Computers in Education, SIIE 2006, vol. 1, pp. 141-148 (2006) 